\title{
Comparison and Evaluation of Methods for Liver Tumor Classification from CT Datasets
}

\author{
S.Gunasundari \\ Dept. of CSE, \\ Velammal Engineering College, Chennai
}

\author{
M. Suganya Ananthi \\ Project Engineer, \\ Wipro Technologies, Bangalore
}

\begin{abstract}
This paper proposes an automatic system for early detection of liver diseases from Computed tomography (CT) images. The general Computer Aided Diagnosis (CAD) system, including liver diagnosis can be done by segmenting a liver and lesion, extracting features and classify disease whether it is hepatoma or hemangioma. To segment a liver from CT abdominal images histogram analyzer and morphological operation is used. Then to extract a lesion from liver Fuzzy c-mean (FCM) clustering is used. In feature extraction biorthogonal wavelet, Gray-level co-occurrence matrix (GLCM) and fast discrete curvelet transform (FDCT) techniques are used. The textural information obtained was used to train various neural network such as Back propagation Neural Network (BPN), Probabilistic Neural Network (PPN) and Cascade feed forward BPN (CFBPN).The outcome obtained from neural networks are compared with each other to find best combination of features and neural network.
\end{abstract}

\section{Keywords}

Morphological Opening and closing, FCM, Probabilistic Neural Network, BPN, CFBPN, FDCT, Biorthogonal wavelet

\section{INTRODUCTION}

The liver is an organ in upper abdomen that serves in digestion and removes waste products from blood. In liver extra waste cells can form a mass of tissue called a growth or tumor. Tumors can be benign or malignant [13]. Benign tumors are not cancer. Usually, doctors can remove them. In most cases, benign tumors do not come back after they are removed. Hemangiomas are benign tumors consisting of masses of twisted, congested blood vessels. Malignant tumors are cancer. Most primary liver cancers begin in hepatocytes (liver cells). This type of cancer is called hepatocellular carcinoma or malignant hepatoma.

The most common medical imaging studies for early detection and diagnosis of liver cancers include ultrasonography (US), computed tomography (CT), magnetic resonance imaging (MRI) [13]. CT is often the preferred method for diagnosing many different cancers, including colon cancer, since the image allows a physician to confirm the presence of a tumor and to measure its size, precise location, and the extent of the tumor's involvement with other nearby tissue. CT examinations can be used to plan and properly administer radiation treatments for tumors, and to guide biopsies and other minimally invasive procedures.
Suhuai Luo, et al. [1] proposed approach of liver segmentation contains three major steps: Texture feature extraction, support vector machine classification, and combined morphological operations. Here liver boundary was segmented manually by radiologist. E-Liang Chen et al. [2] used co-occurrence matrix for extract Haralick et al. [4] texture features. In this approach image is analyzed at only one scale. Mala K. et al. [4] extracted features using Biorthogonal wavelet transform and classified using Probabilistic Neural Network. The drawback of wavelet transform is image have limited directionality and decomposition. Chien-cheng Lee et al. [8] used SVM classifier for classification. They obtained $91 \%$ result in SVM and proved SVM is better than RBF and MLP network. Manual segmentation and gray scale co-occurrence matrix features are used. This paper is organized as follows. Section 2 gives a detailed description of the methods such as Adaptive threshold detection, FDCT, BPN and PPN. Section 3 provides implementation results and section 4 contains a conclusion.

\section{PROPOSED METHOD}

In this paper, we compared each neural network such as BPN, CFBPN, and PPN with various feature extraction methods by evaluating performance of classifiers. Here GLCM, Biorthogonal wavelet and FDCT feature extraction techniques are considered. By comparisons we can identify best feature and neural network combination. System structure is shown in Figure. 1.

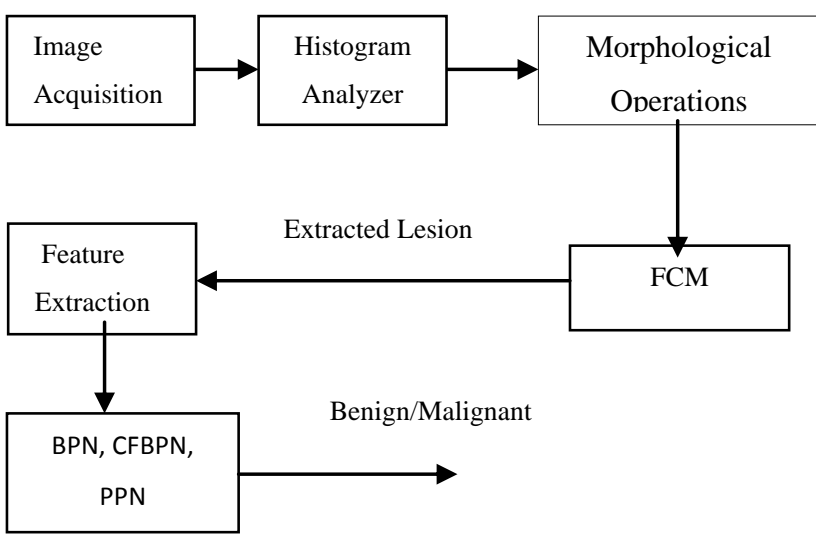

Fig.1. System Structure 


\subsection{Segmentation of Liver}

In general the liver is located on the superior side of the CT abdominal image and takes up the biggest region in a CT image [13]. Fixed threshold technique is not possible for the reason that intensity of CT image differs according to the $\mathrm{CT}$ machine. So the pane size is fixed from a CT image by removing lower portion of CT abdominal image [4]. The histogram is drawn and analyzed for an area inside the pane. The highest pitch represents middle intensity of the liver region and certain intensities are included to accommodate any variance in the liver region pixels. The pixels in the particular range of intensity are extracted [5] [13] [14]. Morphological opening and closing is used to form a liver object from histogram analyzer output. The morphological operations is defined by

$$
f * b=(f \cdot b) \circ b
$$

Where $\mathrm{f}$ is the target image, $\mathrm{b}$ is the structuring element, Means morphological closing ${ }^{\circ}$ Means morphological opening

Obstacles can affect an accurate diagnosis. Histogram Analyzer is used to remove obstacles based on intensity and some obstacles removed by location of liver in CT image. The morphological operations are again applied after removing the obstacles [13]. The image obtained after applying circle structuring element is complemented and multiplied with the original image to get the segmented liver.

\subsection{Segmentation of Lesion}

Fuzzy c-means (FCM) is a method of clustering which allows one piece of data to belong to two or more clusters [4], [13]. Fuzzy c-Means clustering is used to segment the lesion from extracted liver [14]. The maximum Number of iterations specified is 150 . The pixels of the input image are divided into 3 clusters. The first cluster includes pixels in the background. The second cluster includes pixels in the liver other than lesion and the third cluster includes pixels in the lesion [13]. The post processing is done to remove some obstacles. Morphological closing and opening is applied again.

\subsection{Texture Feature Extraction}

Texture is one of the most important defining characteristics of an image. Texture shows its characteristics by both pixel and pixel values. Here FDCT, Biorthogonal wavelet transform, Gray-level co-occurrence matrix are used for texture classification.

\subsubsection{FDCT}

Fast Discrete Curvelet Transform introduced to overcome inherent limitations [2] of multiscale wavelet. The Curvelet transform is a multiscale pyramid with many directions and positions at each length scale, and needle-shaped elements at fine scales. The FDCT has two algorithms such as, curvelet via Unequispaced FFT Transform and curvelet via Wrapping. Wrapping algorithm is better in computation time. Hence wrapping technique is used in this paper. FDCT wrapping implementation [2] FFT of the CT image is obtained. Then separate FFT into collection of Digital Corona tiles. For each corona tile first convert the tile to origin. Then wrap the parallelogram shaped support of tile around a rectangle centered at the origin. The inverse FFT of the wrapped support is taken, and then the curvelet array collection of curvelet coefficients is added. The tumor image is decomposed using subband decomposition $\log 2 \mathrm{M}$ (M size of image) at different scales and angles and real valued curvelet transform applied to extract FDCT coefficient [4]. From FDCT coefficients mean, standard deviation, variance, skewness, kurtosis, entropy, energy, Inverse difference moment (IDM), cluster shade, cluster prominence, information correlation features are extracted. The suitable features are selected and given as input to classifier.

\subsubsection{Gray-level Co-occurrence Matrix}

The Gray Level Co-occurrence Matrix (GLCM) method is a way of extracting second order statistical texture features. The matrix element $\quad P(\mathrm{i}, \mathrm{j} \mid \mathrm{d}, \theta)$ contains the second

order statistical probability values for changes between gray levels $i$ and $j$ at a particular displacement distance $d$ and at a particular angle $(\mathscr{\theta})$. The extracted lesion image pixel pairs co-occurrences are calculated for different distance 1,2,3 etc with different direction such as $0^{\circ}, 45^{\circ}, 90^{\circ}, 135^{\circ}$.

Second order texture features energy, entropy, IDM, Cluster shade, Cluster Prominence, Information correlation are derived from gray-level co-occurrence matrix for classifier.

\subsubsection{Biorthogonal Wavelet Transform}

A biorthogonal wavelet is a wavelet where the associated wavelet transform is invertible but not necessarily orthogonal. Designing biorthogonal wavelets allows more degrees of freedoms than orthogonal wavelets. One additional degree of freedom is the possibility to construct symmetric wavelet functions. In the biorthogonal case, there are two scaling functions $\varphi_{0} \hat{\varphi}$, which may generate

different multiresolution analyses, and accordingly two different wavelet functions $\psi_{0} \widetilde{\psi}$. so the numbers $M, N$ of coefficients in the scaling sequences a, $\widetilde{Q}$ may differ. The scaling sequences must satisfy the following biorthogonality condition,

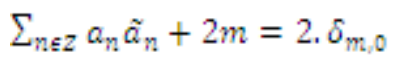

Then the wavelet sequences can be determined as

$$
\begin{aligned}
& b_{n 1}=(-1)^{n} \widetilde{a}_{M-1-n n} n=0, \ldots n-1 \text { and } \\
& \widetilde{b}_{n}=(-1)^{n} \widetilde{Q}_{M-1-n n} n=0, \ldots N-1
\end{aligned}
$$

Biorthogonal wavelet decomposition is applied on lesion image and horizontal, vertical, detailed coefficients are extracted. On that wavelet coefficient gray-level cooccurrence matrix is constructed and statistical features are derived for classification.

\subsection{Classification}

Back propagation Neural Network (BPN) provides better solution in Pattern classification problem. BPN is known as Learning in feed-forward networks in which pairs of input and output values are fed into the network for many cycles, so that the network 'learns' the relationship between the 
input and output. A set of weights are arbitrarily chosen for each processing element. After the input is applied to the first layer of network units, the output is propagated to the next layer as an input to that layer until an output from the network is calculated (output layer). This output pattern is compared to the desired output, and a mean-squared error signal is calculated. An error is created as the sum of the squares of the differences between the each calculated and desired output. The error value is then propagated backwards through the network, and small changes are made to the weights in each layer. The cycle is repeated until the overall error value drops below some predetermined threshold.

The back-propagation algorithm consists of following four steps,

1. This error derivative (EA) is the difference between the actual and the expected action. The rate at which error changes for output changes should be calculated.

$$
E A_{j}=\frac{\partial E}{\partial y_{j}}=y_{j}-d_{j}
$$

2. The rate at which the error changes for the total input received when an output unit is changed should be calculated. This quantity (EI) is the answer from step 1 multiplied by the rate at which the output of a unit changes as its total input is changed.

$$
E I_{j}=\frac{\partial E}{\partial x_{j}}=\frac{\partial E}{\partial y_{j}} \times \frac{d y_{j}}{d x_{j}}=E A_{j} y_{j}\left(1-y_{j}\right)
$$

3. The rate at which the error changes for a weight on the connection into an output unit are changed should be calculated. This quantity (EW) is the answer from step 2 multiplied by the activity level of the unit from which the connection emanates.

$$
E W_{i j}=\frac{\partial E}{\partial W_{i j}}=\frac{\partial E}{\partial x_{j}} \times \frac{\partial x_{j}}{\partial W_{i j}}=E I_{j} y_{j}
$$

4. The rate at which the error changes as the activity of a unit in the previous layer is changed should be calculated. This key step allows back propagation to be applied to multilayer networks. When the activity of a unit in the previous layer changes, it affects activates of all the output units to which it is connected. So to compute the overall effect on the error, we add together all these separate effects on output units. But each effect is simple to calculate. It is the answer in step 2 multiplied by the weight on the connection to that output unit.

$$
E A_{i}=\frac{\partial E}{\partial y_{i}}=\sum_{j} \frac{\partial E}{\partial x_{j}} \times \frac{\partial x_{j}}{\partial y_{i}}=\sum_{j} E I_{j} W_{i j}
$$

By using steps 2 and 4, we can convert the EAs of one layer of units into EAs for the previous layer. This procedure can be repeated to get the EAs for as many previous layers as expected. Once we know the EA of a unit, we can use steps 2 and 3 to compute the EWs on its incoming connections.

CFBPN is like BPN. During back propagation additional neurons are added to hidden layer as needed to improve classification [12].

Probabilistic neural networks (PPN) are forward feed networks built with three layers. They are derived from Bayes Decision Networks. They train quickly since the training is done in one pass of each training vector, rather than several. Probabilistic neural networks estimate the probability density function for each class based on the training samples.

The probabilistic neural network uses a similar probability density function. This is calculated for each test vector. This is what is used in the dot product against the input vector as described below. Usually a spherical Gaussian basis function is used, although many other functions work equally well. Vectors must be normalized prior to input into the network. There is an input unit for each dimension in the vector.

The input layer is fully connected to the hidden layer. The hidden layer has a node for each classification. Each hidden node calculates the dot product of the input vector with a test vector subtracts 1 from it and divides the result by the standard deviation squared. The output layer has a node for each pattern classification. The sum for each hidden node is sent to the output layer and the highest values wins. The Probabilistic neural network trains immediately but execution time is slow and it requires a large amount of space in memory.

The statistical features are given as input to BPN, CFBPN and PPN and disease are distinguished.

\subsection{Performance Evaluation}

A Performance of different networks for various features is evaluated by calculating Accuracy, Sensitivity, Specificity, Positive predictive value (PPV) and Negative predictive value (NPV). Formulas are listed in table I [13] [14]. When combining each neural network such as BPN, PPN and CFBPN with FDCT, different grouping of texture features such as mean, standard deviation, variance, skewness, kurtosis, entropy, energy, IDM, cluster shade, cluster prominence, information correlation etc., is united for FDCT. Best combination of suitable texture features for FDCT selected by using following formulas. Specifically the accuracy value is better in particular selected features compared to other combined features then those features are considered for evaluation. Similarly texture features are chosen for GLCM and biorthogonal wavelet as well. 
TABLE 1

Formulas for evaluating Performance of classifier

\begin{tabular}{|c|c|}
\hline Parameters & Formula \\
\hline Accuracy & $(\mathrm{TP}+\mathrm{TN}) /(\mathrm{TP}+\mathrm{TN}+\mathrm{FP}+\mathrm{FN})$ \\
\hline Sensitivity & $\mathrm{TP} /(\mathrm{TP}+\mathrm{FN})$ \\
\hline Specificity & $\mathrm{TN} /(\mathrm{TN}+\mathrm{FP})$ \\
\hline PPV & $\mathrm{TP} /(\mathrm{TP}+\mathrm{FP})$ \\
\hline $\mathrm{NPV}$ & $\mathrm{TN} /(\mathrm{TN}+\mathrm{FN})$ \\
\hline
\end{tabular}

True Positive (TP): lesions called hepatoma and prove to be hepatoma

False Positive (FP): lesions called hepatoma that proves to be hemangioma

False Negative (FN): lesions that are called hemangioma and prove to be hepatoma

True Negative (TN): lesions that are called hemangioma and prove to be hemangioma

\section{IMPLEMENTED RESULT}

In this work, all CT abdominal images are downloaded from the various sources. Here, Hemangioma considered as benign tumor and hepatoma considered as malignant tumor. Totally 40 hepatoma and 30 hemangioma images are considered for training and testing. Since the liver is located in the left side of the CT image (Figure. 2(a)) the window slice including liver and other obstacles in the window is selected (Figure. 2(b)).

Then the histogram is drawn (Figure. 2(c)) for this window and analyzed. The intensity point with the maximum count is identified. The pixels in the determined range of intensity are extracted. The resultant is the sand like structure (Figure.2 (d)).

After applying threshold, morphological closing and opening are performed to get the object. Structuring element is fixed experimentally. Gray level threshold is applied to the image. The resultant image is complemented. Based on the location information, some obstacles are eliminated. Some obstacles close to the liver boundary with the same intensity, as liver is not eliminated. The objects in the lower right of the image are considered as obstacles and it is removed. After removing the obstacles the morphological closing and opening is again applied to get the liver structure. Through experiment the values for structuring element is fixed. Disk like structuring element is used for both closing and opening. Gray level threshold is applied and the resultant image is complemented. The complemented image is multiplied with the original image to get the liver. (Figure. 2(e)).

To segment the lesion from the liver Fuzzy c-means clustering technique is used. The number of clusters is defined as three. The obstacles in the lesion are removed by applying morphological closing and opening operation. (Figure.2 (f)).

In Table 3 sample segmented result is shown. The extracted lesion is given as input to feature extraction module. The mean, standard deviation, variance, skewness, kurtosis, entropy, energy, IDM, cluster shade, cluster prominence, information correlation are extracted from the tumor image using different feature extraction methods for instance FDCT, Gray level co-occurrence matrix and biorthogonal wavelet transform. Every extracted feature is given to various neural networks such as BPN, CFBPN and PPN. The results Obtained from each neural network are compared with other one to find a best combination of features and neural network.

TABLE 2

Accuracy of Neural Networks

\begin{tabular}{|l|l|l|l|}
\hline $\begin{array}{c}\text { Keatures } \\
\text { Network }\end{array}$ & FDCT & $\begin{array}{c}\text { Gray scale } \\
\text { Co- } \\
\text { occurrence } \\
\text { Matrix } \\
\%\end{array}$ & $\begin{array}{c}\text { Biorthogonal } \\
\text { Wavelet }\end{array}$ \\
\hline BPN & $\mathbf{9 6}$ & $\mathbf{9 6}$ & \% \\
\hline PPN & $\mathbf{9 6}$ & 95.83 & 88 \\
\hline CFBPN & $\mathbf{9 6}$ & 91 & ------- \\
\hline
\end{tabular}

Table 2 reports the classification performance of BPN, CFBPN and PPN for each feature such as FDCT, Gray level co-occurrence Matrix and Biorthogonal Wavelet based on accuracy. Similarly sensitivity, specificity, PPV and NPV as well compared.

FDCT coefficients are derived using FDCT via wrapping. Tumor image may be decomposed for different angles and scales. The angles can be changed in multiples of four. Here, the tumor image decomposed using three scales and angle used is 16. From extracted coefficients mean, standard deviation, variance, skewness, kurtosis, entropy, energy, IDM, cluster shade, cluster prominence, information correlation are calculated and given as input to each classifier. The kurtosis, skewness, energy, information correlation, inverse difference moment, entropy feature combinations performed better in BPN,PPN and CFBPN neural networks with $96 \%$.

Gray scale co-occurrence matrix is extracted from tumor image and extracted features are fed as input to every neural network. It is performed well in BPN with accuracy $96 \%$, sensitivity $100 \%$, specificity $90 \%$, PPV 93\%, NPV $100 \%$.PPN and CFBPN accuracy results are less compared with BPN. 


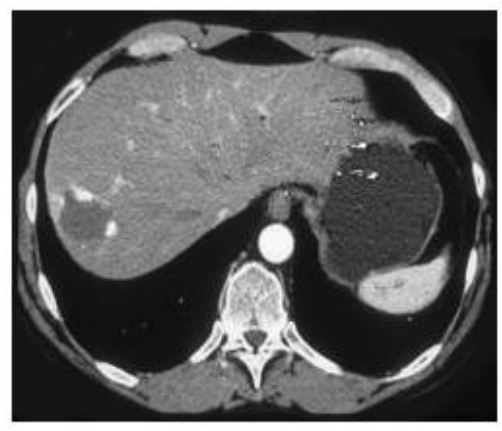

(a) CT image

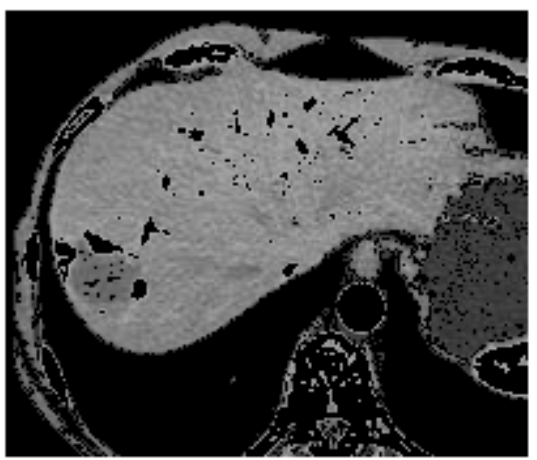

(d) Threshold Image

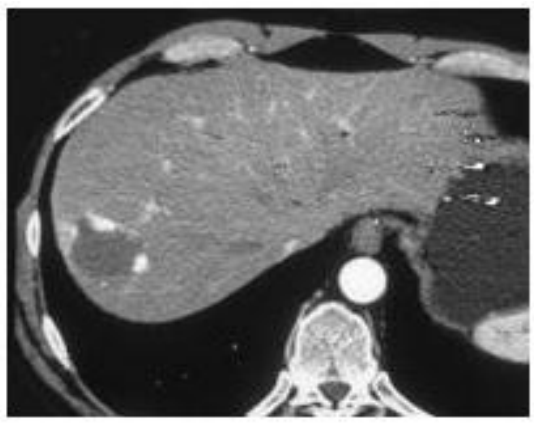

(b) Window slice

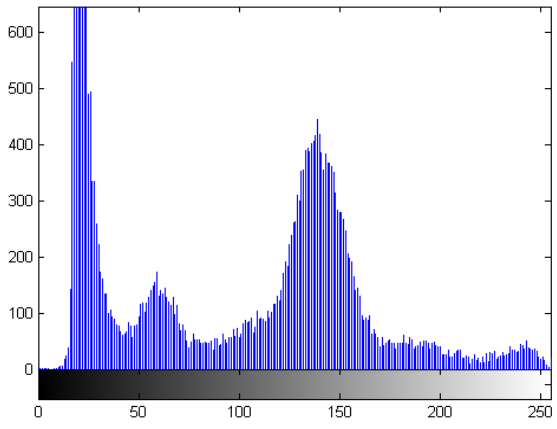

(c) Histogram

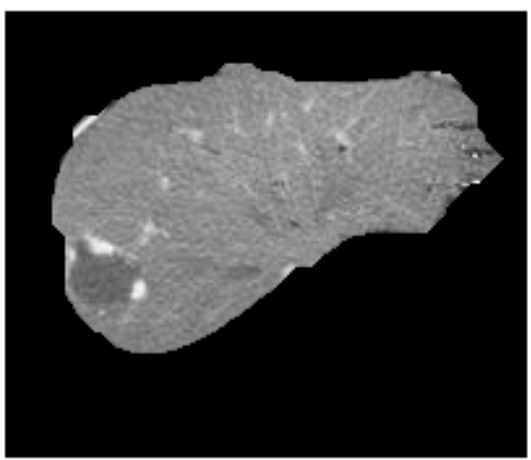

(e) Segmented Livers
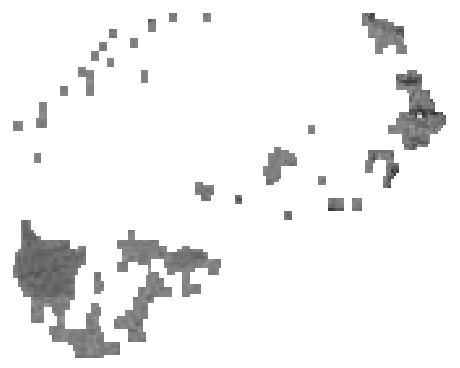

(f) Segmented Lesions

TABLE 3

Segmented Liver and Lesion

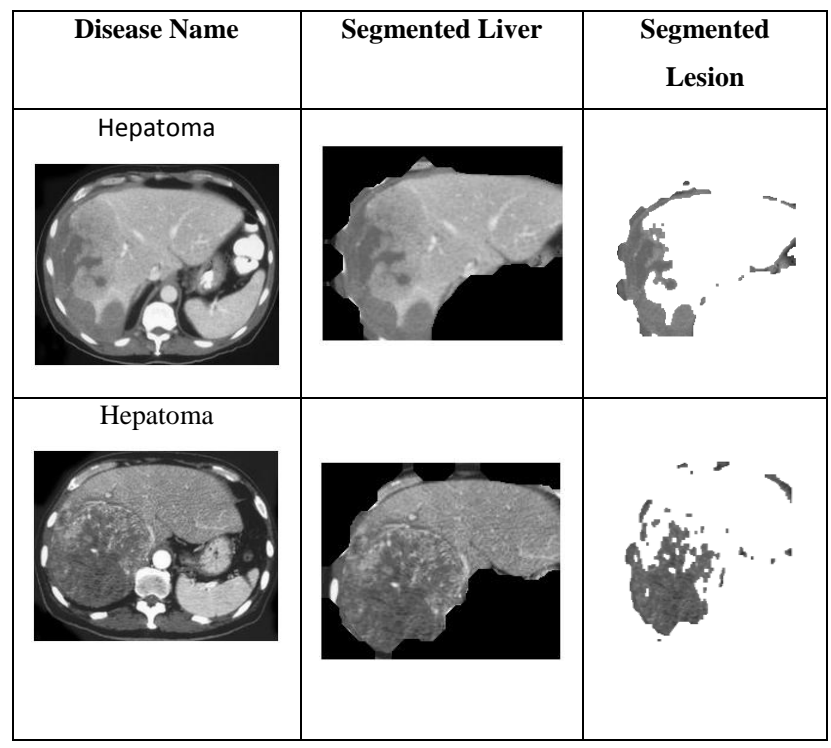

Hepatoma


Biorthogonal wavelet transform bior1.3 yields better outcome than other wavelets. The tumor image is decomposed using bior 1.3 and extracted wavelet coefficient gray scale co-occurrence matrix is applied to extract second order statistical features such as entropy, energy, IDM, cluster shade, cluster prominence, information correlation. Then cluster shade, cluster prominence, mean feature combination yields considerable result $88 \%$ accuracy,94\% sensitivity, $88 \%$ PPV, $88 \%$ NPV is achieved on PPN neural network. BPN and CFBPN achieved only less than 50\% value.Two third of total images are given as training set and remaining one third of them given as test set.

\section{CONCLUSION}

The proposed CAD system is used to segment the lesion using adaptive threshold, morphological closing and opening and Fuzzy C-means clustering. An evaluation of the segmentation quality shows well output by visual results. The segmentation algorithm proves to be simple, but effective. In comparisons, FDCT yields better result in all neural networks. Gray scale co-occurrence matrix performed well in BPN and Biorthogonal wavelet worked well in PPN. In future, it will be tested on great number of images and different type of images.

\section{REFERENCES}

[1] Suhuai Luo, Jesse S. Jin, Stephan K.Chalup, Guoyu Qian,"A Liver Segmentation Algorithm Based on Wavelets and Machine Learning", International Conference on Computational Intelligence and Natural Computing, 2009.

[2] Candes, E., Demanet, L., Donoho, D., \& Ying, L. "Fast Discrete Curvelet Transforms", Technical Report, Cal Tech, March 2006.

[3] E-Liang Chen, Pau-CHoo Chung, Ching-Liang Chen, Hong-Ming Tsai and Chein I Chang, "An Automatic Diagnostic system for CT Liver Image Classification", IEEE Transactions Biomedical Engineering, vol 45, no. 6, pp. 783-794, June 1998.

[4] S.S. Kumar, Dr R.S.Moni, "Diagnosis of Liver Tumor from CT Images Using Fast Discrete Curvelet Transform", IJCA Special Issue on "Computer Aided Soft Computing Techniques for Imaging and Biomedical Applications", 2010
[5] K.Mala, V.Sadasivam, and S.Alagappan," Neural Network based Texture Analysis of Liver Tumor from Computed Tomography Images" International Journal of Biological and Life Sciences, 2006.

[6] Pavlopoulos.S, Kyriacou.E, Koutsouris.D, Blekas.K, Stafylopatis. A, Zoumpoulis.P, "Fuzzy Neural Network-Based Texture Analysis of Ultrasonic Images," IEEE Engineering in Medicine and Biology, pp. 39-47, Feb 2000

[7] Robert.M, Haralick, K.Shanmugam,Dinstein, "Texture Features for Image Classification", IEEE Transactions on Systems, Man, and Cybernetics, Vol.SMC-3, No. 6, pp 610-621, Nov. 1973.

[8] Chien-Cheng Lee, Yu-Chun Chiang, Chun-Li Tsai, and Sz-Han Chen,"Distinction of Liver Disease from CT images using Kernel-based Classifiers, ICMED, Vol.1, No.2, Page113-120, March 2007.

[9] A.K. Jain, "Fundamentals of Digital Image Processing", Prentice-Hall International Editions, N.J., USA, 1989.

[10] Rafael C.Gonzalez and Richard E.Woods," Digital Image Processing",Addison-Wesley Publishing Company, 1993.

[11] Satish Kumar, "Neural Networks: a classroom approach “, TATA McGraw-Hill, 2010.

[12] S. Gunasundari, S. Baskar, "Application of Artificial Neural Network in identification of Lung Diseases", world congress on Nature \& Biologically Inspired Computing, 2009.

[13] Mala, K., Sadasivam, V., "Wavelet based texture analysis of Liver tumor from Computed Tomography images for characterization using Linear Vector Quantization Neural Network", International Conference on Advanced Computing and Communication,2006.

[14] Mala, K., Sadasivam, V., "Automatic Segmentation and Classification of Diffused Liver Diseases using Wavelet Based Texture Analysis and Neural Network", INDICON, 2005 